\title{
Energy expenditure estimates of the Caltrac accelerometer for running, race walking, and stepping
}

\author{
Pamela D Swan, William C Byrnes, Emily M Haymes
}

\section{Department of Exercise Science and Physical Education, Arizona State University, Tempe, AZ 85287-0701, USA P D Swan}

Department of Kinesiology, University of Colorado, Boulder, Colorado, USA W C Byrnes

Department of Nutrition, Food and Movement Sciences, Florida State University, Tallahassee, FL, USA E M Haymes

Correspondence to: Dr P D Swan, Department of Exercise Science and Physical Education, Box 870701, Arizona State University, Tempe, AZ 85287-0701, USA.

Accepted for publication 3 April 1997

\begin{abstract}
Objective-To examine the accuracy of the Caltrac accelerometer for estimating energy expenditure (EE) during three exercise modes.

Methods-A subset of 31 women (mean (SEM) age 22.6 (5) years) was selected from a training study comparing various physiological parameters during race walking, running, and stepping. Subjects each performed mode specific graded exercise tests to peak $\dot{V}_{2}$. Regression equations for $\dot{V}_{2} v$ heart rate (HR) were generated from each individual's test data. EE (kcal and $\mathrm{kJ}$ ) was estimated for each $\dot{\mathrm{V}}_{2}$ value based on the respiratory exchange ratio, and kcal $v \mathbf{H R}$ regression equations were generated to predict $\mathrm{EE}$ from each subject's HR data (EE-HR). HR in the field was monitored by telemetry, and two Caltrac monitors, one set for EE and one to give counts, were attached to a belt over opposite hips.
\end{abstract}

Results-EE-HR was not significantly different across exercise modes. Caltrac overestimated EE $(P<0.01)$ in runners (14\%) and walkers (19\%) but underestimated EE in steppers by about $10 \%(P=$ 0.12).

Conclusions-The Caltrac is a reliable instrument but it did not accurately distinguish $E E$ in running, race walking, or stepping in a group of young women. (Br 7 Sports Med 1997;31:235-239)

Keywords: energy cost; women; stride frequency; exercise

The progressive attempts to link increased physical activity with positive health benefits have intensified the need for the development of instruments or methods that can easily, inexpensively, and accurately quantify energy expenditure (EE) in the population. ${ }^{12}$ The Caltrac (Hemokinetics, Inc) accelerometer is a small lightweight microcomputer which, when programmed with age, gender, height, and mass, is used to measure EE of free ranging individuals. ${ }^{3-6}$ Worn on a belt over the hip, the Caltrac calculates EE based on vertical acceleration measurements of the body. The Caltrac is manufactured to be sensitive primarily to the frequency and magnitude of vertical accelerations and decelerations. ${ }^{78}$ Previous studies have shown the Caltrac to be fairly reliable for some types of dynamic activities ${ }^{3}$ and to be quite accurate for estimates of EE during normal walking. ${ }^{10}$ However, the overall effectiveness of the Caltrac for estimating $\mathrm{EE}$ has been questioned because it may not be able to accurately distinguish and then compensate for variations in intensity of some activities. ${ }^{711} 12$

Increasing the intensity of an activity requires various techniques depending upon the type of activity. For example, in race walking, intensity is increased by increasing the frequency of strides, whereas in running, intensity is primarily elevated by increasing the stride length. ${ }^{13}{ }^{14}$ Furthermore, stepping exercise normally requires the maintenance of a set frequency-that is, music or metronome beat - and thus added intensity is developed by increasing arm movements or the magnitudethat is, stepping height-of the vertical displacement.

Currently race walking and stepping exercise modes are becoming more popular as alternative techniques to give variety in exercise routines and/or change the pattern by which exercise intensity is increased. The ability to examine $E E$ in free ranging individuals performing various types of physical activities is desirable to help further the understanding of the association between physical activity and the prevention of hypokinetic disease. No previous studies could be found in the literature that assessed the accuracy of the Caltrac in free moving environments such as during race walking or stepping exercise. We hypothesised that, because of the different movement patterns of each exercise mode, the Caltrac would be most sensitive to exercises that link intensity to vertical acceleration such as stepping or race walking as compared with running. Thus the purpose of this study was to examine the accuracy of the Caltrac accelerometer for estimating EE during three modes of exercise in a field setting.

\section{Methods}

The 31 participants (10 runners, 10 steppers and 11 race walkers) in this study were a subset of volunteers selected from a training study comparing various physiological parameters during race walking, running, and stepping in college-aged (mean (SEM) age $22.6(0.8)$ years) women. Testing took place during the last six weeks of the 28 week training study. Initially, instruction was given in each mode of training, and the subjects were randomly assigned as runners, steppers, or race walkers. All subjects were very practised and proficient 
in their assigned exercise mode before participation in this investigation. The subjects were informed verbally and in writing of the procedures involved in the study and gave their written consent to participate. All procedures in this study were approved by the University Committee on Human Research.

The overall training routine required each subject to exercise four days a week for 40 minutes at $80 \%$ of her maximal heart rate (HR). Testing consisted of attaching two Caltrac monitors to a belt at the waist over opposite hips. One unit was programmed with the subject's age, height, mass, and gender to compute EE based on the constants contained in the Caltrac microcomputer. The other Caltrac was programmed to give activity counts $(\mathrm{CNTS})^{15}$ where age $=99$, mass $=25$, height $=$ 36 , and sex $=0$, thereby turning off the computer's calculation of EE but maintaining the electronic raw data generated by the Caltrac microchip. Three to five subjects in each group were selected to be tested each day while the others continued with their daily training schedule. Each subject was tested twice (within 7-14 days) during the six week investigation.

Before testing, height and mass were measured and the participants were asked to stretch and "warm up". Each was fitted with a Polar (Polar CIC, Inc.) HR monitor. Immediately before starting the 40 minute exercise bout, the heart watch stopwatch was started and the two Caltrac monitors were simultaneously set to zero by two investigators. Exercise HR was monitored and data were stored every minute in the heart watch microcomputer.

The runners and race walkers were instructed to follow the same route (outside on paved pathway) each time they were tested. All were told to keep their exercise intensity within their target $\mathrm{HR}$ range (previously determined to be $70-80 \%$ of $\mathrm{HR} \max$ ) throughout the exercise bout. The steppers remained in the gymnasium and participated in a "step aerobics" class led by a certified professional exercise leader. All the movements and music were designed for "low impact" high intensity exercise to help the participants to meet their HR goal. The steppers had previously determined their individual appropriate step height for the routine. The routine was essentially "canned" in that the music beat and movements were the same (but in a different order) in each class period. Immediately after the 40 minute bout (stepping, race walking, or running), investigators simultaneously read and recorded the Caltrac monitor's digital display and stopped the stop watch.

Subjects participating in the study each performed mode specific - that is, race walking, running, and stepping-graded exercise tests (GXTs) to peak aerobic capacity ( $\dot{\mathrm{V}}_{2}$ peak) at the conclusion of the study. The walking test required subjects to race walk at $4.0 \mathrm{mph}$ on a motor driven treadmill at a $0 \%$ grade for two minutes. Every two minutes, speed was increased by $0.5 \mathrm{mph}$ until the subject reached an $\mathrm{HR}$ of 160 beats/min. At this point the grade of the treadmill was increased by $2.0 \%$ until the subject reached volitional exhaustion. The running test was similar to the walking tests, although the initial speed was set at $4.0 \mathrm{mph}$ at $0 \%$ grade for two minutes. Again speed was increased by $0.5 \mathrm{mph}$ every two minutes until an HR of 160 beats/min was achieved. At this point the grade was again increased by $2.0 \%$ until exhaustion. The stepping test required subjects to step at a rate of 120 steps per minute on a 4 inch step for two minutes. Every two minutes, the stepping rate was increased by 20 steps per minute until an HR of 160 beats/ min was reached at which point the step height was increased by 2 inches every two minutes until exhaustion. During each test HR was monitored by a three lead electrocardiograph. Electrodes were placed on the chest in a modified V5 lead before exercise. HR was monitored continuously throughout the GXT and recorded using an $\mathrm{E}$ for $\mathrm{M}$ (Electronics for Medicine, Boulder, CO, USA) three channel electrocardiograph during the last ten seconds of each minute of exercise.

Metabolic measures were determined every 30 seconds during each GXT utilising a computer assisted open circuit indirect calorimetry system with appropriate metabolic software. Expired gas was collected continuously using an Applied Electrochemistry S-3A oxygen analyser and a Beckman LB-2 carbon dioxide analyser. Inspired minute ventilation was measured with a Hans Rudolph pulmonary pneumotachometer. The system was calibrated over the expected ranges of inspired volumes and gas fractions. Gas analysers were calibrated before exercise by three concentrations of calibration gas previously determined by a certified standard gas accurate to $0.01 \%$. Metabolic measures were considered maximal if there was no rise in oxygen consumption with increasing work load or if the respiratory exchange ratio was greater than 1.15 and the peak HR was within 10 beats/min of the subject's age predicted maximal HR.

Regression equations for $\dot{\mathrm{VO}}_{2}$ versus $\mathrm{HR}$ were generated from each individual's appropriate (mode specific) GXT data. To determine the individual $\mathrm{HR}-\mathrm{V}_{2}$ regression line, at least five calibration points were obtained during the last 30 seconds of each two minute stage by simultaneous measurement of $\dot{\mathrm{VO}}_{2}$ and recording of $\mathrm{HR}$. EE was estimated for each $\dot{\mathrm{V}}_{2}$ value based on the respiratory exchange ratio for each exercise stage. ${ }^{16}$ Individual kcal $v$ $\mathrm{HR}$ regression equations were generated to predict EE from each subject's $H R$ data (EE-HR).

During the 40 minute exercise in the field, five-minute $H R$ intervals were averaged from the HR monitor and then used in the regression equation to ensure the most representative estimate of the EE across the time period. The EE value predicted in this way (EE-HR) was used as the criterion with which the Caltrac EE (EE-CT) was compared. Because Caltrac estimated EE is strongly influenced by subject body mass but CNTS values are not, we also evaluated exercise EE by adjusting estimated EE by each subject's individual body mass (EE-HR/KG and EE- 
Table 1 Physical characteristics (values are mean (SEM))

\begin{tabular}{lllll}
\hline & Runner $(n=10)$ & Stepper $(n=10)$ & Walker $(n=11)$ & Population $(n=31)$ \\
\hline Age (years) & $20.1(0.8)$ & $23.7(1.7)$ & $23.8(1.6)$ & $22.6(0.8)$ \\
Height $(\mathrm{cm})$ & $164.3(1.8)$ & $166.9(2.0)$ & $165.4(1.8)$ & $165.6(1.0)$ \\
Mass $(\mathrm{kg})$ & $62.8(2.6)$ & $66.7(5.3)$ & $62.6(2.5)$ & $64.0(2.0)$ \\
$\dot{\text { Vo }}{ }_{2}$ peak $(\mathrm{ml} / \mathrm{kg} / \mathrm{min})$ & $42.5(1.6)^{\star}$ & $37.3(1.3)$ & $36.4(1.5)$ & $38.7(1.0)$ \\
\hline
\end{tabular}

$\star \mathrm{P}<0.05$, runner $v$ walker.

$\mathrm{CT} / \mathrm{KG}$ ) to correlate with Caltrac activity counts (CNTS).

Analysis of variance was used to obtain group descriptive data and to compare EE-CT with EE-HR. Comparisons between trials were carried out by dependent $t$ tests. Comparisons between modes were made by repeated measures analysis of variance. Pearson product correlation coefficients and linear regression equations were determined between variables and between trials. Statistical analyses were performed by a Macintosh microcomputer using a Statview 512+ statistical package.

\section{Results}

Table 1 gives the physical characteristics of the subjects. No significant differences were found between groups except in the mode specific $\dot{\mathrm{V}}_{2}$ peak values. After training, the runners were able to obtain a higher value than the walkers. A strong linear relationship (range $r=$ $0.92-0.99$ ) was found between $\dot{\mathrm{Vo}}_{2}$ and $\mathrm{HR}$ for each individual during graded exercise for each exercise mode.

Table 2 Comparison of energy expenditure and heart rate between trials. (Values are mean (SEM))

\begin{tabular}{lllll}
\hline Group & Trial A & Trial B & Correlation (r) & $\begin{array}{l}\text { Significance }(P \\
\text { value) }\end{array}$ \\
\hline $\begin{array}{llll}\text { HR (beats/min) } \\
\quad \text { Runner }\end{array}$ & $165.7(1.4)$ & $167.0(1.7)$ & 0.87 & $<0.01$ \\
$\quad \begin{array}{l}\text { Stepper } \\
\text { Walker }\end{array}$ & $160.9(4.6)$ & $161.7(4.7)$ & 0.88 & $<0.01$ \\
EE-HR (kJ) & $157.3(1.1)$ & $155.0(1.2)$ & 0.68 & $<0.05$ \\
$\quad$ Runner & $1449.3(45.6)$ & $1503.7(62.8)$ & 0.85 & $<0.01$ \\
$\quad$ Stepper & $1315.0(124.3)$ & $1307.9(118.4)$ & 0.96 & $<0.01$ \\
$\quad$ Walker & $1183.2(44.8)$ & $1190.3(49.0)$ & 0.88 & $<0.01$ \\
EE-CT (kJ) & & & & $<0.01$ \\
$\quad$ Runner & $1646.4(37.2)$ & $1678.2(58.2)$ & 0.86 & $<0.01$ \\
$\quad$ Stepper & $1178.6(112.1)$ & $1099.6(124.3)$ & 0.98 & $<0.05$ \\
$\quad$ Walker & $1412.1(62.3)$ & $1425.5(54.0)$ & 0.78 & $<0.01$ \\
CNTS & & & & $<0.05$ \\
$\quad$ Runner & $62.9(1.6)$ & $63.2(2.4)$ & 0.85 & $<0.01$ \\
$\quad$ Stepper & $39.3(2.0)$ & $40.1(2.3)$ & 0.79 & 0.92 \\
$\quad$ Walker & $55.2(3.6)$ & $54.2(4.2)$ & & \\
\hline
\end{tabular}

$\mathrm{EE}=$ energy expenditure; $\mathrm{HR}=$ heart rate; CNTS = activity counts from Caltrac accelerometer $\mathrm{EE}-\mathrm{HR}=$ energy expenditure from $\mathrm{HR} v \mathrm{~V}_{2}$ regression equation; EE-CT = energy expenditure from Caltrac accelerometer.

Table 3 Comparison of energy expenditure and activity counts between groups. (Values are mean (SEM))

\begin{tabular}{llll}
\hline & Runner $(n=10)$ & Stepper $(n=10)$ & Walker $(n=11)$ \\
\hline EE-HR (kcal) & $346.4(10.9)$ & $314.3(29.7)$ & $282.8(10.7)$ \\
$\quad(\mathrm{kJ})$ & $1449.3(45.6)$ & $1315.0(124.3)$ & $1183.2(44.8)$ \\
EE-HR/KG (kcal/kg) & $5.6(0.2)^{\mathrm{a}}$ & $4.7(0.3)$ & $4.5(0.1)$ \\
$\quad(\mathrm{kJ} / \mathrm{kg})$ & $23.3(0.9)$ & $19.3(1.3)$ & $19.0(0.5)$ \\
EE-CT (kcal) & $393.5(8.9)^{\mathrm{b}}$ & $281.7(26.8)$ & $337.5(14.9)$ \\
$\quad(\mathrm{kJ})$ & $1646.4(37.2)$ & $1178.6(112.1)$ & $1412.1(62.3)$ \\
EE-CT/KG (kcal/kg) & $6.3(0.2)^{\mathrm{b}}$ & $4.3(0.2)$ & $5.5(0.3)$ \\
$\quad(\mathrm{kJ} / \mathrm{kg})$ & $26.5(1.0)$ & $17.8(0.9)$ & $22.8(1.2)$ \\
$\mathrm{CNTS}$ & $62.9(1.6)^{\mathrm{b}}$ & $39.3(2.0)^{\mathrm{c}}$ & $55.2(3.6)$ \\
\hline
\end{tabular}

${ }^{\mathrm{P}} \mathrm{P}<0.05$, runner $v$ walker.

${ }^{\mathrm{b}} \mathrm{P}<0.05$, runner $v$ stepper.

${ }^{c} \mathrm{P}<0.05$, walker $v$ stepper.

EE-HR = energy expenditure from heart rate $v \dot{\mathrm{V}} \mathrm{O}_{2}$ regression equation; EE-HR/KG = mass adjusted energy expenditure from heart rate $v \dot{\mathrm{V}}_{2}$ regression equation; $\mathrm{EE}-\mathrm{CT}=$ energy expenditure from Caltrac accelerometer; $\mathrm{EE}-\mathrm{CT} / \mathrm{KG}=$ mass adjusted energy expenditure from Caltrac accelerometer; CNTS = activity counts from Caltrac accelerometer.
Table 2 indicates the comparison between trials by group. Mean values and the test-retest correlation coefficients from the 40 minute exercise bouts are shown. No significant differences in HR, EE-HR, EE-CT, or CNTS were found between trials for any group. Also the reliability was high between trials. Because of the overall high correlation between trials, only trial A data were used in the remainder of the analyses.

Group analyses (table 3 ) showed no significant differences $(P>0.05)$ in the criterion estimate of EE between exercise mode. However, group differences $(P<0.01)$ were noted in EE-CT. EE-CT during running was significantly greater $(P<0.05)$ than EE-CT for stepping. Caltrac significantly overestimated $(P<0.01) \mathrm{EE}$ in running by about $14 \%$ and race walking by about $19 \%$. Although the Caltrac EE underestimated stepping by about $10 \%$, values were not significantly different from the criterion in stepping $(P=0.12)$. When EE data were adjusted for body mass, a different pattern emerged. Specifically, the EE-HR/KG data were lower $(P<0.05)$ in walkers than runners.

Table 4 indicates moderate but significant relationships between EE-HR and EE-CT for both steppers $(r=0.78)$ and walkers $(r=0.60)$ but the relationship $(r=0.58)$ did not meet statistical significance $(P=0.08)$ for runners. The correlation between EE-CT/KG and CNTS was fairly high for each group. However, the correlation between the EE-HR/KG and CNTS was only statistically significant ( $P$ $=0.05$ ) for race walking.

\section{Discussion}

The purpose of this study was to assess the accuracy of the Caltrac accelerometer for estimating EE during three different modes of exercise. The findings confirmed that the Caltrac personal activity computer was reliable in providing a value for $\mathrm{EE}$ regardless of exercise mode, but it significantly overestimated EE for running (14\%) and race walking (19\%) activities, and underestimated EE for stepping $(10 \%)$ compared with the HR $v$ EE criterion estimate. There was a moderate relationship between the EE estimates between the criterion HR-EE regression equation method and the

Table 4 Correlations of energy expenditure between groups

\begin{tabular}{lllll}
\hline Group & $r$ & $R^{2}$ & $S E E$ & $\begin{array}{l}\text { Significance } \\
(P \text { value })\end{array}$ \\
\hline Runner & & & & \\
EE-HR $v$ EE-CT & 0.58 & 0.34 & 1.02 & 0.08 \\
EE-HR/KG $v$ CNTS & 0.45 & 0.20 & 0.65 & 0.19 \\
EE-CT/KG $v$ CNTS & 0.78 & 0.61 & 0.47 & $<0.01$ \\
Stepper & & & & \\
EE-HR $v$ EE-CT & 0.78 & 0.61 & 0.60 & $<0.01$ \\
EE-HR/KG $v$ CNTS & 0.20 & 0.04 & 1.03 & 0.58 \\
EE-CT/KG $v$ CNTS & 0.63 & 0.40 & 0.58 & 0.05 \\
Walker & & & & \\
EE-HR $v$ EE-CT & 0.60 & 0.36 & 1.20 & 0.05 \\
EE-HR/KG $v$ CNTS & 0.60 & 0.36 & 0.35 & 0.05 \\
EE-CT/KG $v$ CNTS & 0.89 & 0.79 & 0.44 & $<0.01$ \\
\hline
\end{tabular}

$\mathrm{EE}-\mathrm{HR}=$ energy expenditure from heart rate $v \mathrm{~V}_{\mathrm{O}_{2}}$ regression equation; EE-HR/KG = mass adjusted energy expenditure from heart rate $v \dot{V O}_{2}$ regression equation; EE-CT = energy expenditure from Caltrac accelerometer; EE-CT/KG $=$ mass adjusted ture from Caltrac accelerometer; EE-CT/KG = mass adjusted
energy expenditure from Caltrac accelerometer; CNTS = activity counts from Caltrac accelerometer. 
Caltrac estimated EE. Although the results of this study support previously reported findings, this study is unique in that it utilises subjects in free moving environments. The Caltrac was found to be able to estimate the EE of women performing running, stepping, and race walking exercise in a field setting.

The assumption of linearity between $\mathrm{HR}$ and $\mathrm{EE}$ has been previously shown to be valid during walking, running, and cycling. ${ }^{17}{ }^{18}$ Booyens and Hervey ${ }^{19}$ suggested that if EE was to be predicted from HR, the regression line should be derived from activities as similar as possible to those to be measured. At low activity levels or during activities where $\mathrm{HR}$ is influenced by emotion, diet, temperature, previous work, or fatigue ${ }^{18}$ this relationship is suspect. In the current study, data were collected during moderate intensity exercise when the subjects were previously rested, at least four hours after eating and under controlled conditions of temperature. Also each regression line was derived for each subject individually for each exercise mode. The high correlation between the HR response and oxygen consumption for each mode of exercise supported our use of individual regression equations as criterion $\mathrm{EE}$ estimations. Thus despite the indirect nature of the criterion estimate, we were confident that the $\mathrm{HR}-\mathrm{VO}_{2}$ regression equations allowed us to closely estimate the actual EE during each exercise bout.

The Caltrac is manufactured to be sensitive primarily to the frequency and magnitude of vertical accelerations and decelerations. ${ }^{78}$ In this study the mass adjusted EE data supported the suggestion that the EE-CT was related to the CNTS data regardless of exercise mode. This suggests that the EE-CT values are responsive to the accelerometer counts output. However, mean mass adjusted EE-HR data (EE-HR/KG) were different between runners and walkers even though $\mathrm{HR}$ intensities during exercise were similar. The EE-CT/KG data followed the same pattern as the unadjusted EE data (table 3). These results give evidence that efficiency of movement was different between the exercise modes.

The prototype accelerometer device was calibrated to the frequency and vertical accelerations of a walking speed of $3 \mathrm{mph}$. If acceleration is increased more than the energy cost (such as in running), an overestimation of EE by the device will occur. ${ }^{7}$ However, activities that involve lifting the body, such as stepping, have a large EE compared with acceleration, and may result in an underestimation of $\mathrm{EE}$. Indeed, Servais $e t a l^{\prime}$ found that, although the energy cost of climbing ten flights of stairs slowly or rapidly was the same, the accelerometer device measured quite different values. They found the accelerometer to underestimate stair climbing by a factor of 3.1 because the body was not accelerating in proportion to the EE of the activity. Different activities are known to require various methods of increasing EE. In running, after a certain stride frequency has been established, runners will primarily increase speed by increasing the stride length with little change in stride frequency. ${ }^{131420}$ The participants in this study were running (jogging) at an approximate mean speed of $5 \mathrm{mph}$ for the 40 minute exercise bout. Earlier reports have shown that the Caltrac device significantly overestimates EE for running between 4 and $7 \mathrm{mph}^{7{ }^{12}}{ }^{21}$ Walters and Brodie ${ }^{21}$ recently reported that a similar device (Kenz Calorie Counter) was also insensitive to variations in jogging speed brought about by increasing stride length. Thus the results from this study confirm that the programming of accelerometer devices is not particularly responsive to differences in the EE brought about by increased body lift after a certain velocity has been achieved.

In race walking, once maximal stride length has been achieved, intensity is gained by increasing the frequency of strides rather than by increasing body lift. ${ }^{22}{ }^{23}$ With this in mind, we expected that the Caltrac would be able to accurately distinguish EE because the amount of acceleration of the body would be proportional to the amount of EE for the movement. The results from this study showed that the Caltrac overestimated EE for race walking by about $19 \%$. However, this overestimate may be due to the Caltrac's response to the higher stride frequency in race walking. For example, in a study ${ }^{24}$ examining the relationship between stride frequency, CT-EE and CNTS, stride frequency was found to correlate with Caltrac CNTS significantly $(r=0.94)$ in running and normal walking across all speeds. Only when stride frequency was increased was the Caltrac able to adequately discriminate between speeds. $^{24}$

The accelerometer worn at the hip should measure acceleration in the vertical axis. ${ }^{7}$ Perhaps the location of the device (over the hip) influenced the results. Force platform analysis has shown that, in activities such as bench stepping and normal walking, the major acceleration component is in the vertical axis of the earth's gravitational field. ${ }^{7}$ In race walking the major acceleration component may not be in the vertical axis. ${ }^{23}$ Although there are measurable changes in the frequency of steps in race walking, conceivably there is also a greater amount of body rotation which may be detected by the Caltrac causing an overestimation of EE. In this study the mass adjusted EE in race walking, but not in running or stepping, was moderately correlated with CNTS. This may mean that the Caltrac is sensitive to the overall movement patterns of race walkingthat is, stride frequency changes - but that it is also affected by the body rotation patterns of the race walking movement in its CNTS value, resulting in an overestimate of EE. In elite women race walkers, Lafortune et $l^{3}{ }^{3}$ reported on page 17 that during maximal controlled velocity the subjects attempted to "reduce the up and down movement of their body" and that "less energy was expended for vertical movements at the expense of horizontal movements". Thus perhaps a better estimate of EE in race walking would be gained by measuring acceleration at a different location of the body or with a triaxial device. 
In order to be valid and useful as an activity monitor, instruments or devices must be able to quantify EE accurately and be able to distinguish between different types and intensities of activity. This study joins others in substantiating that the Caltrac is a reliable instrument. ${ }^{.10}$ However, the Caltrac personal activity monitor was shown to be able to only estimate EE for running, race walking, or stepping in young women. Results from this study suggest that the Caltrac is relatively insensitive in determining individual EE or discriminating energy cost for modes of activity that require different styles of movement patterns. Perhaps when novel movement patterns such as race walking are studied, an accelerometer that is responsive in three dimensions such as the Tritrac 3RD (Hemokinetics, Inc.) may be more appropriate. However, the correlational data support the continued use of the Caltrac in free moving settings when large population data samples are collected especially if an intensity correction factor such as heart rate could be incorporated.

Grateful recognition is given to graduate students, Heather Silverman, John Noble, and Carol Walker, who assisted in the collection of the data. Partial financial support for this project was provided by a grant from Reebok International L.T.D.

1 Ainsworth B, Montoye HJ, Leon AS. Methods of assessing physical activity during leisure and work. In: Bouchard C, health: international proceedings and consensus statement.

2 LaPorte RE, Montoye HJ, Casperson CJ. Assessment of physical activity in epidemiologic research: problems and

3 Ballor DL, Burke LM, Knudson DV, Olson JR, Montoye HJ. Comparison of three methods of estimating energy expenditure: Caltrac, heart rate, and video analysis. Res $Q$ Exerc Sport 1989;60:362-8.

4 Bray MS, Morrow JR, Pivarnik JM, Bricker JT. Caltrac validity for estimating caloric expenditure with children. Pediatr Exerc Sci 1992;4:166-79.

5 Freedson P, Widrick J, Mazziotti J. Validity of the Caltrac accelerometer in estimating energy expenditure during walking. In: Hermans GPH, ed. Sports, medicine and health. New York: Elsevier Science Publishers, 1990: 620-4.
6 Maliszewski AF, Freedson PS, Ebbeling CJ, Crussemeyer J, Kastango KB. Validity of the Caltrac accelerometer in estimating energy expenditure and activity in children and mating energy expenditure and activity

7 Servais SB, Webster JG, Montoye HJ. Estimating human energy expenditure using an accelerometer device. fournal of Clinical Engineering 1984:9;159-70.

8 Wong TC, Webster JG, Montoye HJ, Washburn R. Portable accelerometer device for measuring human energy expenditure. IEEE Trans Biomed Eng 1981;BME-28:467-71.

9 Montoye HJ, Washburn R, Servais S, Ertl A, Webster JG, Nagel FJ. Estimation of energy expenditure by a portable accelerometer. Med Sci Sports Exerc 1983;15:403-7.

10 Pambianco G, Wing RR, Robertson R. Accuracy and reliability of the Caltrac accelerometer for estimating

11 Balogun JA, Martin DA, Clendenin MA. Calorimetric validation of the Caltrac accelerometer during level walking. Phys Ther 1989;69:501-9.

12 Haymes EM, Byrnes WC. Walking and running energy expenditure estimated by Caltrac and indirect calorimetry. Med Sci Sports Exerc 1993;25:1365-9.

13 Cavanagh PR, Kram R. Stride length in distance running: velocity, body dimensions, and added mass effects. Med Sci Sports Exerc 1989;21:467-79.

14 Hogberg $P$. Length of stride, stride frequency, "flight" period and maximum distance between the feet during running with different speeds. Arbeitsphysiologie 1952;14: 431-6.

15 Sallis JF, Buono MJ, Roby JJ, Carlson D, Nelson JA. The Caltrac accelerometer as a physical activity monitor for school-age children. Med Sci Sports Exerc 1990;22:698703.

16 Weir JB. New methods for calculating metabolic rate with special reference to protein metabolism. $\mathcal{F}$ Physiol (Lond) 1949;109:1-9.

17 Meijer GA, Westerterp KR, Koper H, Hoor FT. Assessment of energy expenditure by recording heart rate and body acceleration. Med Sci Sports Exerc 1989;21:343-7.

18 Acheson KJ, Campbell IT, Edholm OG, Miller DS, Stock MJ. The measurement of daily energy expenditure: an MJ. The measurement of daily energy expenditure: an evaluation

19 Booyens J, Hervey GE. The pulse rate as a means of measuring metabolic rate in man. Can $f$ Biochem Physiol 1960;38:1301-9.

20 Sinning WE, Forsyth HL. Lower-limb actions while running at different velocities. Med Sci Sports Exerc 1970;2: 28-34.

21 Walters NJ, Brodie DA. An evaluation of the Kenz calorie counter during progressive treadmill exercise in adults and children. Pediatric Exercise Science 1996;8:156-65.

22 Cairns MA, Burdett RG, Pisciotta JC, Simon R. A biomechanical analysis of racewalking gait. Med Sci Sports Exerc 1986;18:446-53.

23 Lafortune M, Cochrane A, Wright A. Selected biomechanical parameters of race walking. EXCEL 1989;5:15-17.

24 Haymes EM, Lee DT, Martinez LR. Relationship of stride frequency and Caltrac estimated energy expenditure during walking and running. Int $\mathcal{F}$ Sports Med 1993;14:106 (Abstr).

\section{Commentary}

This carefully executed and well presented piece of work describes the accuracy (reliability and validity) of a commercially available motion sensor, the Caltrac, in measuring energy expenditure during different modes of exercise. The authors conclude that the Caltrac is highly reliable but not particularly valid, and may significantly overestimate (running and walking) or underestimate (stepping) energy expenditure. It is best used to discriminate broad bands of physical activity in large population studies.

C BOREHAM 